[8] - Independence and homomorphisms in abstract algebras, Fundamenta Mathematicae 50 (1961), p. 45-61.

[9] W. Narkiewicz, Independence in a certain class of abstract algebras, ibidem 50 (1962), p. $333-340$.

[10] S. Pickert, Einführung in die höhere Algebra, Göttingen 1951.

[11] J. Schmidt, Einige grundlegende Begriffe und sätze aus der T'heorie der Hüllenoperatoren, Bericht über mathematische Tagung, Berlin 1953, p. $21-48$.

[12] - Mehrstufige Austausohstrukturen, Zeitschrift für mathematische logik und Grundlagen der Mathematik 2 (1956), p. 233-249.

Regu par la Rédaction le 1\%.10. 1961.

\section{MAXIMALE UNTERSEMIGRUPPEN \\ UND KONVEXITÄT IN GRUPPEN}

VON

S. GEADYSZ (WROCEAW)

Die gewöhnliche Definition der Konvexität, hier lineare Konvexität genannt, läßt sich nicht direkt aus linearen Räumen auf Gruppen übertragen. Um eine solche Verallgemeinerung tatsächlich zu erhalten, kann man aber folgende Bemerkung benutzen:

In lokalkonvexen reellen linearen Räumen sind abgeschlossene konvexe Mengen Durchschnitte von abgeschlossenen Stützhalbräumen, können daher gruppentheoretisch und zwar als Durchschnitte von Translationen abgeschlossener maximaler. Untersemigruppen gekennzeichnet werden.

Diese Bemerkung legt schon eine Definition der Konvexität in Gruppen nahe (s. 4). Die beiden Konvexitätsbegriffe, der übliche (lineare Konvexität) und der gruppentheoretische, stimmen für borelsche Mengen in lokalkonvexen linearen Räumen überein (s. 4). Es stellt sich weiter erwartungsgemä $\beta$ heraus, da $\beta$ in kompakten zusammenhängenden Gruppen keine konvexe borelsche Menge außer der Gruppe selbst vorhanden ist (s. 5).

Der gruppentheoretische Konvexitätsbegriff erlaubt die meisten Eigenschaften der konvexen Mengen auf diejenigen von maximalen Untersemigruppen zurückzuführen, es ist daher zweckmäßig diese Semigruppen näher zu betrachten.

Es ergibt sich, daß in topologischen Gruppen jede in bezug auf die inneren Automorphismen invariante (d.h. normale) maximale Untersemigruppe, die eine borelsche Menge ist, abgeschlossen sein $\operatorname{muB}$ (s. 3).

In Abschnitten 1 und 2 wird die Definition der maximalen Untersemigruppe $M$ nebst ihren einfachsten Eingeschaften und einer vollen Charakterisierung der Faktorgruppe $G / M^{*}$ angegeben, wo $M^{*}=M \frown M^{-1}$ gesetzt wird (Satz 1).

1. Maximale Untersemigruppen. Eine Untersemigruppe (USG) $M$ einer abstrakten Gruppe $G$ soll maximal heißen (max USG), wenn 
$\{x, M\}=G$ für jedes $x \notin M$ gilt, wobei $\{A\}$ die von $A$ erzeugte Semigruppe bedeutet.

Jede Gruppe $G$ besitzt eine max USG, nämlich die triviale, welche mit der ganzen Gruppe $G$ zusammenfällt. Es gibt Gruppen, die sonst keine max USG enthalten, z. B. die Gruppen $C_{p}$. Anderseits enthält jede endliche Gruppe eine echte max USG, die gleichzeitig eine maximale Untergruppe ist, eine Untergruppe also, für welche bei jedem $x \notin H$ $\left\{x, x^{-1}, H\right\}=G$ gilt. Eine normale maximale Untergruppe ist zugleich eine max USG. In der Tat: die Falktorgruppe $G / H$ kann keine echte, vom Einselement $e$ verschiedene Untergruppe enthalten. $G / H$ ist also eine endliche zyklische Gruppe und hat man $x \notin H$, so fällt schon die Semigruppe $\{x, H\}$ mit der ganzen Gruppe $G$ zusammen. Daher muß $x^{-1} \epsilon\{x, H\}$ sein, was $\{x, H\}=\left\{x, x^{-1}, H\right\}=G$ für $x \notin H$ ergibt.

Im Folgenden setzen wir eins für alle Mal voraus, daß $G$ aus mehr als einem Element besteht.

Ist $N \subset G$ eine USG, so wird $N^{*}$ die größte in $N$ enthaltene Gruppe bezeichnen. Man hat also $N^{*}=N \cap N^{-1}$. Ist $N$ eine max USG, so ist dieser Durchschnitt nicht leer. Das ergibt sich sofort aus dem

Hilfssatz 1.1. Ist $M$ eine max $U S G$, so gilt $e \in M$.

Beweis. Wäre es $e \notin M$, so hätte $\operatorname{man}\{e, M\}=\{e\} \cup M=G^{*}$, was sofort $M=G \backslash\{e\}$ ergibt. Für jedes $x \in M$ folgt dann $x^{-1} \in M$ und, da $M$ nicht leer ist, $e=x \cdot x^{-1} \in M$.

Man kann leicht folgende zwei Hilfssätze beweisen:

HILFSSATZ 1.2. Ist $p$ ein Homomorphismus von $G$ auf $G^{\prime}$, so ist das Bild und das volle Urbild einer max USG eine max USG.

HILFSSATZ 1.3. Es sei $M C G$ eine echte normale max USG und $p$ der natürtiche Homomorphismus $\varphi: G \rightarrow G^{\prime}=G / M^{*}$. gilt

Dann ist $M^{\prime}=\varphi(M)$ eine echte normale max $U S G$ in $G^{\prime}$ und dabe

und

$$
\varphi^{-1}\left(M^{\prime}\right)=\varphi^{-1}(\varphi(M))=M
$$

wo $e^{\prime}$ das Einselement in $G^{\prime}$ bezeichnet.

Wir wollen noch bemerken, daß $M^{*}$ ein Normalteiler in $G$ ist, wenn nur $M$ eine normale max USG ist.

2. Die Faktorgruppe $G / M^{*}$. Ist $M$ eine normale max USG, so hat die Faktorgruppe $G^{\prime}=G / M^{*}$ erwartungsgemä $\beta$ eine besonders einfache Struktur (s. Satz 1).

Hilfssatz 2.1. Ist $M$ eine normale max $U S G$ und ist $x_{0}^{-1} \& M$, so gibt es eine natürliche Zahl $n_{0}$, für welche $x_{0}^{n_{0}} \in M$ ist.

Beweis. $M$ ist maximal und daher gilt $\left\{x_{0}^{-1}, M Y\right\}=G$. Da $M$ dabei normal ist, so kann man $x_{0}$ in der Gestalt

$$
x_{0}=x_{0}^{-k} \cdot g, \quad g \in M, \quad k \geqslant 0,
$$

darstellen, was $x_{0}^{k+1} \epsilon M$ ergibt.

HILfssatz 2.2. $M$ sei eine nomale max USG. Gibt es ein $x_{0}$ mit $x_{0}$, $x_{0}^{-1} \& M$, so existiert ein ganzes $n>0$ derart, $d a \beta x^{n} \in M$ für jedes $x$ aus $G$ gilt.

Beweis. Es ist $\left\{x_{0}, M\right\}=G$ und so läßt jedes $x \in G$ eine Darstellung

$$
x=g \cdot x_{0}^{n(x)}, \quad g \in M, \quad n(x) \geqslant 0,
$$

zu. Für $n_{0}$ erhält man aus Hilfssatz 2.1

$$
x^{n_{0}}=h \cdot x_{0}^{n_{0} \cdot n(x)}, \quad h \in M .
$$

Es ist dann offenbar $x^{n_{0}} \in M$.

Jetzt sei $N$ eine USG von $G$, für die folgende zwei Bedingungen erfüllt sind:

(2.1) Für jedes $x \in G$ ist entweder $x \in N$ oder $x^{-1} \in N$.

(2.2) Ist $x \in N$ und zugleich $x^{-1} \in N$, so ist $x=e$.

Dann definiert die Relation

$$
x \leqslant y \equiv y \cdot x^{-1} \in N
$$

eine rechtsinvariante Ordnung, denn man hat:

(i) Es gilt $x \leqslant y$ oder $y \leqslant x$ für jedes Paar $x, y \in G$.

(ii) Aus $x \leqslant y$ und $y \leqslant x$ folgt $x=y$.

(iii) Ist $x \leqslant y$ und $y \leqslant z$, so ist $x \leqslant z$.

(iv') Aus $x \leqslant y$ folgt $x z \leqslant y z$ für jedes $z \epsilon G$.

HILFSSATZ 2.3. Diese Ordnung ist dann und nur dann beiderseits invariant, wenn $N$ normal ist (s. z. B. [3], S. 538).

Ist nun $N$ normal, so gilt auch

(iv') Aus $x \leqslant y$ folgt $z x \leqslant z y$ für jedes $z \epsilon G$.

Daraus schließt man

(v) Ist $x \leqslant y$ und $z \leqslant t$, so gilt $x z \leqslant y t$.

(vi) Ist $x \leqslant y$, so gilt $y^{-1} \leqslant x^{-1}$.

HILFSSATZ 2.4. Ist $N$ eine normale max $U S G$, so ist die Ordnung (2.3) archimedisch: für jedes Paar $x, y>$ e gibt es eine natüliche Zahl $k$ derart, $d a \beta x \leqslant y^{k}$ ist. 
Beweis. Man kann sich auf $e<y<x$ beschränken. Hat man $y^{k}<x$ für jedes ganze $k>0$, so ist auch, nach (vi),

$$
x^{-1}<y^{-k}, \quad k>0 \text {. }
$$

Aus $y>e$, aus (2.1) and (2.2) ergibt sich unmittelbar $y^{-1} \notin N$ und, da $N$ maximal ist, so haben wir $\left\{y^{-1}, N\right\}=G$. Man hat also $x^{-1}=g \cdot y^{-n}$, $g \in N$, wobei $n>0$ ist, weil $x^{-1} \notin N$ gilt. (v) liefert weiter: $x^{-1} \geqslant e \cdot y^{-n}$ $=y^{-n}$, was aber im. Widerspruch zu (2.4) steht.

Wir sind jetzt imstande den Hauptsatz über normale maximale Untersemigruppen in abstrakten Gruppen zu beweisen.

SaTz 1. Ist $M$ eine echte normale max $U S G$ der Gruppe $G$, so ist die Faktorgruppe $G^{\prime}=G \mid M^{*}$ abelsch, und zwar: falls $M^{*}=M$, so ist $G^{\prime}$ zylkisch von Primzahlordnung, falls aber $M^{*} \neq M$, so ist $G^{\prime}$ einer Untergruppe. der additiven Gruppe der reellen Zahlen isomorph.

Beweis. $\varphi$ sei der natürliche Homomorphismus von $G$ auf: $G^{\prime}$. Nach Hilfssatz 1.3 ist die Semigruppe $M^{\prime}=\varphi(M)$ eine echte max USG in $G^{\prime}$.

Wir werden zwei Fälle unterscheiden:

$1^{0}$ Es gibt ein $x_{0} \in G^{\prime}$ mit $x_{0}, x_{0}^{-1} \notin M^{\prime}$.

$2^{\circ}$ Für jedes $x \in G^{\prime}, x \neq e^{\prime}$, gehört von den Elementen $x$ and $x^{-1}$ genau eines zu $M^{\prime}$.

Das erschöpft schon alle Möglichkeiten, weil nach (1.1) die beiden Relationen $x \in M^{\prime}$ und $x^{-1} \in M^{\prime}$ für $x \neq \iota^{\prime}$ nicht gleichzeitig bestehen können.

In dem ersten Falle gewährleistet Hilfssatz 2.2 die Existenz eines ganzen $n>0$ derart, da $\beta x^{n} \in M^{\prime}$ für $x \in G^{\prime}$ gilt. Daraus folgt sofort, da $\beta$ $x^{-1}=x^{-n} \cdot x^{n-1} \in M^{\prime}$, wenn nur $x \in M^{\prime}$ ist. Also enthält $M^{\prime}$ zusammen mit $x$ die ganze zyklische Gruppe $\left\{x, x^{-1}\right\}$. Zugleich muß aber $\left\{x, x^{-1}\right\}$ $C M^{\prime *}$ sein, was, nach (1.1), $x=e^{\prime}$ ergibt. Die ganze maximale USG $M^{\prime}$ reduziert sich also auf die triviale Untergruppe $\left\{e^{\prime}\right\}$. Daher kann die Gruppe $G^{\prime}$ keine nichttrivialen echten Untergruppen besitzen, mithin ist sie endlich und zyklisch von Primzahlordnung. Das ist nur dann. möglich, wenn $M^{\prime}=M^{\prime *}=\left\{e^{\prime}\right\}$, also wenn $M=M^{*}$ ist.

Im zweiten Falle (dann ist sicher $M^{*} \neq M$ ), können wir in $Q^{\prime}$ oine Ordnung vermöge (2.3) definieren. Nach Filfssatz 2.3 und. 2.4 ist diese Ordnung beiderseits invariant und archimedisch. Eine so geordnete Gruppo ist aber einer Gruppe von reellen Zahlen isomorph (s. z. B. [1.], s. 9 u. If.).

Man kann Satz 1 umkehren. Diese Umkehrung liegt auf der Hand und ist grundsätzlich bereits im Hilfssatz 1.2 mitenthaltien.

3. Max USG in topologischen Gruppen. Indem wir zu den topologischen Gruppen übergehen, setzen, wir uns zum. Ziel, die topologi- sche Struktur der maximalen Untersemigruppen zu untersuchen, die sich als besonders einfach erweist (s. Satz 2).

$G$ sei jetzt eine lokalkompakte topologische Gruppe mit dem linksinvarianten Haarschen Maß $m$.

HILFSSATZ 3.1. Wenn in einer lokalkompakten Gruppe $G$ auch nur eine borelsche normale max $U S G M$ existiert, so ist $G \sigma$-kompakt und es gilt $m(M)>0$.

Der Beweis stützt sich auf die Bemerkung, daß wenn $M$ eine solche Semigruppe ist, dann $G=\bigcup_{n=1}^{\infty} x^{n} M$ für jedes $x \notin M$ gilt, und daß eine borelsche Menge $\sigma$-kompakt ist.

HiLfSSATZ 3.2. $M$ sei eine borelsche Menge und $N$ eine dichte Menge in einer o-kompakten Gruppe $G$.

$$
\text { Die Gleichheit }
$$

$$
m(x M \backslash M)=0 \quad \text { für } \quad x \in N
$$

ist nur dann möglich, wenn $m(M)=0$ oder $m(G \backslash M)=0$ ist.

Beweis. Wir werden zuerst zeigen, daß das Maß der symmetrischen Differenz $x M-M$ verschwindet:

$$
m(x M-M)=0, \quad x \in G .
$$

Aus (3.1) und aus der Stetigkeit der Funktion

$$
f_{U}(x)=m((x M \backslash M) \frown U)
$$

für kompakte Mengen $U$, folgt, daß $m(x M \backslash M)=0$ für $x \in G$ gilt. Um (3.2) zu beweisen, genügt es zu zeigen, daß auch

$$
m(M \backslash x M)=0 \quad \text { für } \quad x \in G
$$

ist.

Wäre das nicht der Fall, so würde ein $x_{0}$ mit $m\left(M \backslash x_{0} \cdot M\right)>0$ und eine kompakte Menge $V$ existieren, für welche

$$
\begin{aligned}
m\left(\left(x_{0}^{-1} M \backslash M\right) \cap V\right) & =m\left(x_{0}^{-1}\left(\left(M \backslash x_{0} M\right) \cap x_{0} V\right)\right)= \\
& =m\left(\left(M \backslash x_{0} M\right) \frown x_{0} V\right)>0 .
\end{aligned}
$$

Für $x_{n} \rightarrow x_{0}^{-1}, x_{n} \in N$, erhält man jetzt aus (3.1) und aus der Stetigkeit der Funktion (3.3) für $U=x_{0} V$

$$
\begin{aligned}
0 & =m((x M \backslash M) \cap V) \rightarrow m\left(\left(x_{0}^{-1} M \backslash M\right) \cap V\right)= \\
& =m\left(\left(M \backslash x_{0} M\right) \frown x_{0} V\right)>0 .
\end{aligned}
$$

Dieser Widerspruch bestätigt (3.4) und zugleich (3.2).

$\chi$ sei die charakteristische Funktion der Menge $M$. Da wegen (3.2)

$$
m\left(x^{-1} M-M\right)=\int_{\Theta}[\chi(x y)-\chi(y)]^{2} d y=0
$$


für jedes $x$ gilt, so, gilt auch $\chi(x y)=\chi(y)$ für jedes $x$ bei $m$-fast allen $y$. Wegen des Fubinischen Satzes gibt es dann ein $y_{0}$ derart, daß die Gleichung

$$
\chi\left(x y_{0}\right)=\chi\left(y_{0}\right)
$$

für fast alle $x$ besteht. Der Hilfssatz ist also bewiesen.

SATZ 2. In lokalkompakten Gruppen sind borelsohe normale max USG abgeşchlossene Mengen.

Beweis. $M$ sei eine solche max USG und $\bar{M}$ ihre abgeschlossene Hülle. Augenseheinlich muß $\bar{M}=M$ oder $\bar{M}=G$ sein. Es genügt nur den zweiten Fall zu betrachten. Wir werden zeigen, da $\$$ dann $M=Q$ ist.

Nach. Hilfssatz 3.1 ist $G \sigma$-kompakt und dabei gilt auf einer dichten Menge (für $x \in M$ nämlich) $x M \subset M$, was offenbar (3.1.) ergibt. Mann kann. also Hilfssatz 3.2 anwenden, woher man $m(M)=0$ oder $m(G \backslash M)=0$ erhält. Man hat aber, nochmals wegen Hilfssatz 3.1, $m(M)>0$ und daher $m(G \backslash M)=0$. Da für das Haarsche Maß $m, m\left(A^{-1}\right)=0$ mit $m(A)=0$ gleichbedeutend ist, so hat man auch:

$$
m\left(G \backslash M^{-1}\right)=m\left((G \backslash M)^{-1}\right)=m(G \backslash M)=0 .
$$

Mithin haben wir endlich

$$
m\left(G \backslash M^{*}\right)=m\left(G \backslash M \cap M^{-1}\right)=0,
$$

woher schon $M^{*}=G$ und umsomehr $M=Q^{*}$ folgt. Gäbe es nämlich ein $x_{0} \notin M^{*}$, so wäre es $x_{0} M^{*} \cap M^{*}=\varnothing$; das steht aber im Widerspruch zu (3.5), weil $m\left(x M^{*}\right)=m\left(M^{*}\right)$ ist.

Satz 2 ist also bewiesen.

4. Konvexe Mengen in Gruppen. Wir werden in einer topologischen Gruppe $G$ mit $\mathscr{K}$ die kleinste Familie von. Untermengen bezeichnen, für welche folgende drei Bedingungen erfüllt sind:

I. $\mathscr{K}$ enthält alle abgeschlossenen normalen $\max$ USG

II. Ist $K \in \mathscr{K}$, so ist auch $x K, K x \in \mathscr{K}, x \in G$

III. Es ist $\bigcap_{a} K_{\alpha} \epsilon \mathscr{K}$, wenn $K_{r \alpha} \epsilon \mathscr{K}$.

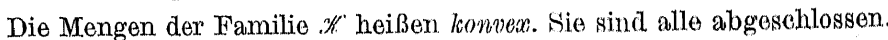
Wir werden jetzt diesen Begriff mit dem der linearen Konvexität vergleichen (s. Satz 3).

Ist $X$ ein reeller linearer Raum, so heißt die Untermenge $K$ linear konvex, wenn

$$
t x+\tau y \in K
$$

wo $t, \tau \geqslant 0, t+\tau=1, x, y \in K$. Es ist zweckmäßig hier nur abgeschlossene Mengen zu betrachten.
HILFSSATZ 4.1. Ist $M$ eine normale max USG einer abstrakten Gruppe $G$ und ist $H$ eine Untergruppe von $G$, so ist $N=M \cap H$ eine max USG in $H$.

Beweis. Es ist zu zeigen, daß

$$
\{x, N\}=H, \quad \text { wenn nur } \quad x \notin N, x \in H .
$$

Wir beweisen das indirekt. Es gelte also für ein $y$

$$
y \notin\{x, N\}, \quad y \in H \text {. }
$$

Da $x \notin M$, so ist $\{x, M\}=G$ und daher gibt es ein $g \in M$, für welches $y=x^{k} g$ ist. Das ergibt aber $g=x^{-k} y \in H \cap-M=N$, was zu (4.1) im Widerspruch steht.

HImfssatz 4.2. Die additive Gruppe $R_{1}$ der reellen Zahlen, mit der gewöhnlichen Topologie, hat nur zwei echte abgeschlossene max USG: $[0, \infty)$ und $(-\infty, 0]$.

Beweis. Es genügt zu zeigen, daß für abgeschlossene max USG $M$ die Bedingung $M \cap(0, \infty) \neq \varnothing$ schon $M \cap(-\infty, 0)=\emptyset$ ergibt. Daraus folgt: $M \subset[0, \infty)$ und, da $M$ maximal ist, auch $M=[0, \infty)$.

Nach Hilfssatz 3.1 ist das Lebesguesche Maß von $M$ positiv. Wäre es also $M \cap(0, \infty) \neq \varnothing$ und dabei $M \cap(-\infty, 0) \neq \varnothing$, so gäbe es sicher in $M$ zwei Zahlen $x$ und $y$ mit irrationalem Verhältnis und von verschiedenem Vorzeichen. Da aber die Punkte $k x+l y, k, l=1,2, \ldots$, eine dichte Menge in $R_{1}$ bilden und da $M$ abgeschlossen ist, so muß $M=R_{1}$ sein. Das widerspricht aber der Voraussetzung, daß $M$ eine echte Untermenge ist.

SATZ 3. In reellen linearen lokalkonvexen Räumen sind die konvexen Mengen genau die linear konvexen und abgeschlossenen Mengen.

Beweis. Wir werden zuerst beweisen, daß eine konvexe (und somit abgeschlossene) Menge $M$, eine linear konvexe Menge ist. Wir können uns dabei nur auf max USG beschränken.

Für irgendwelche zwei Punkte $x, y \in M$ setzen wir:

$$
\pi_{x}=\{z: t x\}, \quad \pi_{y}=\{z: t y\}, \quad-\infty<t<\infty .
$$

Die Mengen $\pi_{x}$ und $\pi_{y}$ sind offenbar Geraden oder auch einzelne Punkte, das letztere falls $x=0$ oder $y=0 . \pi_{x}$ und $\pi_{y}$ sind Untergruppen und deshalb sind die Mengen $M_{x}=M \curvearrowleft \pi_{x}, M_{y}=M \cap \pi_{y}$ auf Grund von Hilfssatz 4.1 max USG in $\pi_{x}$ bzw. in $\pi_{y}$. Diese Mengen sind abgeschlossen in der auf $\pi_{x}$ und $\pi_{y}$ bezogenen Topologie des betrachteten Raumes. Diese induzierte Topologie stimmt aber für lokalkonvexe Räume mit der gewöhnlichen überein.

Man kann also Hilfssatz 4.2 anwenden und sofort

$$
t x \in M_{x} \subset M, \quad \tau y \in M_{y} \subset M \text { für. } t, \tau \geqslant 0
$$

Colloquium Mathematicum IX. 
erhalten, da $x \in M_{x}$ und $y \in M_{y}$ ist. $M$ ist aber eine Semigruppe, was $t x+\tau y \in M$ ergibt. Das ist aber bereits die verlangte lineare Konvexität von $M$.

Es sei nun $K$ eine abgeschlossene linear konvexe Menge. Ist $x_{0} \notin K$, so gibt es bekanntlich eine Hyperebene, die $x_{0}$ von. $K$ trennt. Genauer, es gibt ein lineares (stetiges) Funktional $F(x)$ and eine Zahl a mit

$$
F(x)\left\{\begin{array}{lll}
\geqslant a & \text { für } & x \in K, \\
<a & \text { für } & x=x_{0} .
\end{array}\right.
$$

Demnach ist die Menge $K$ ein Durchschnitt von. TIalbräumen von der Gestalt

$$
N=\{x \mid F(x) \geqslant a\} .
$$

Es genügt also zu zeigen, daß (4.2) konvexe Mengen sind.

Das ergibt sich aber aus der Bemerkung, daß im Falle $F(y)=a$ die Menge

$$
N_{y}=N-y=\{x \mid F(x) \geqslant 0\}
$$

eine abgeschlossene max USG, also eine konvexe Menge ist. Somit ist $N$, als Translation einer konvexen Menge, selbst konvex.

5. Kompakte Gruppen. Was andere (nichtlineare) Gruppen an. betrifft, so ist der Sachverhalt in den zusammenhängenden kompakten Gruppen besonders einfach und klar (s. Satz 4).

Wir werden uns auf folgenden Satz von Koch und Wallace stützen ([4], Satz 4.3): Wenn $S$ eine kompakte Semigruppe mit $S=S \cdot S$ ist, und wenn es in $S$ genau ein idempotentes Element gibt, so ist $S$ eine Gruppe.

Jetzt sei $G$ eine kompakte Gruppe und $m$ das Haarsche Maß in $G$. Wir beginnen mit

HILFSSATZ 5.1. Der Index einer abgeschlossenen normalen max USG $M$ in einer kompakten Gruppe $G$ ist gleich

$$
n=m(G) / m(M)<\infty .
$$

Beweis. Die Voraussetzungen des Satzes von. Koch und Wallace sind hier für $S=M$ erfüllt, wobei das Einselement der Gruppe $Q$ die Rolle des idempotenten Elements spielt (Hilfssatz 1.1). So haben wir uns überzeugt, daß $M$ eine Untergruppe der Gruppe $G$ und dabei von positivem Maße ist (Hilfssatz 3.1).

Um (5.1) zu erhalten, genügt es jetzt die Gruppe $G$ in disjunkte mit $M$ kongruente Nebenklassen zu zerlegen.

SATz 4. In einer kompakten zusammenhängenden Gruppe gibt es keine eohte abgesohlossene normale max USG.
Oder in der Sprache der Konvexität: die einzige konvexe Menge in einer kompakten zusammenhängenden Gruppe $G$ ist die Gruppe G selbst.

Der Beweis ergibt sich unmittelbar aus Hilfssatz 5.1: in einer zusammenhängenden Gruppe ist der Index entweder unendlich oder 1 . Da er endlich sein $\mathrm{mu} B$, so ist $M=G$.

Es ist noch bemerkenswert, $\mathrm{da} \beta$ in einer kompakten zusammenhängenden Gruppe unmeßbare max USG existieren können, wie zum Beispiel die Gesamtheit der nichtnegativen Zahlen in der additiven Gruppe der reellen Zahlen mit einer kompakten und zusammenhängenden Topologie (vgl. z. B. [2], S. 182).

Dagegen ist die Frage nach der Existenz abgeschlossener echter nichtnormaler max USG in kompakten zusammenhängenden Gruppen ungelöst.

\section{LITERATURNAOHWEIS}

[1] N. Bourbaki, Topologie générale, chapitres V-VIII, Paris 1955.

[2] S. Hartman und C. Ryll-Nardzewski, Zur Theorie der lokalkompakten abelschen Gruppen, Colloquium Mathematicum 4 (1957), S. 157-188.

[3] Е. С. Л япин, Полуаруппи, Москва 1960.

[4] A. D. Wallace, The structure of topological semigroups, Bulletin of the American Mathematical Society 61 (1955), S. 95-112.

Reçu par la Rédaction le 1.7.1961 